\title{
Linfoma Ileal Primário como uma Causa de Intussuscepção Ileocecal Recorrente
}

\author{
Primary Ileal Lymphoma as a Cause of Recurrent Ileocecal
}

\author{
FÁBIOCÉSAR MIRANDATORRICELLI'; ROBERTO IGLESIAS LOPES ${ }^{2}$; ANDRÉ RONCON DIAS ${ }^{3}$; GIOVANNI SCALA \\ MARCHINI $^{4}$; WANDERLEY WESLEY BONAFÉ5; JULIANA MAGALHÃES LOPES ${ }^{6}$; MARCELO RODRIGUES BORBA ${ }^{7}$
}

\author{
1. Médico Residente do Departamento de Cirurgia Geral do Hospital das Clínicas da Faculdade de Medicina da \\ Universidade de São Paulo (HC-FMUSP); ${ }^{2}$ Médico Residente do Departamento de Urologia do HC-FMUSP; ${ }^{3}$ Médico \\ Residente do Departamento de Cirurgia do Aparelho Digestório do HC-FMUSP; ${ }^{4}$ Médico Residente do Departamento \\ de Cirurgia Geral do HC-FMUSP, ${ }^{5 .}$ Interno do $6^{\circ}$ ano da Faculdade de Medicina da Universidade de São Paulo; \\ 6. Médica Residente do Departamento de Coloproctologia do Hospital Heliopólis, São Paulo; ${ }^{7}$. Médico Assistente do \\ Departamento de Cirurgia Geral do Hospital Universitário da Universidade de São Paulo - SP.
}

TORRICELLI FCM; LOPES RI; DIAS AR; MARCHINI GS; BONAFÉ WW; LOPES JM; BORBA MR. Linfoma Ileal Primário como uma Causa de Intussuscepção Ileocecal Recorrente. Rev bras Coloproct, 2008;28(2): 246-250.

RESUMO: Introdução: A intussuscepção ocorre quando um segmento proximal do intestino invagina para dentro do lúmen do segmento distal adjacente. Esta patologia é relativamente comum em crianças, sendo geralmente idiopática, diferentemente do que é evidenciado em adolescentes e adultos, os quais apresentam uma causa orgânica comprovada na maioria dos casos. $O$ linfoma intestinal como etiologia desta patologia é extremamente raro. Relato de Caso: Um paciente de 16 anos, masculino, referindo dor abdominal em quadrante inferior direito há $\mathbf{3 6}$ horas associada a vômitos e fezes com sangue vivo compareceu em nosso serviço. $O$ exame físico se apresentava dentro da normalidade exceto por uma massa palpável no quadrante inferior direito. A ultra-sonografia abdominal revelou intussuscepção ileocecal. A colonoscopia demonstrou uma massa protuberante proveniente do orifício da válvula ileocecal que foi reduzida, tendo o paciente um alívio completo dos sintomas. Três semanas após, o paciente retornou ao nosso hospital com recorrência dos sintomas. Uma laparotomia exploradora foi realizada evidenciando uma massa polipóide no íleo terminal com intussuscepção para dentro do ceco. Uma colectomia direita ampliada foi realizada. Após exame patológico da peça e estadiamento tumoral, um linfoma de Burkitt primário foi diagnosticado. A recuperação pós-operatória não apresentou intercorrências e o paciente foi encaminhado para quimioterapia adjuvante.

Descritores: Linfoma. Intussuscepção. Íleo. Imuno-histoquímica. Neoplasias.

\section{INTRODUÇÃO}

A intussuscepção ocorre quando um segmento proximal do intestino invagina para dentro do lúmen do segmento distal adjacente. Congestão venosa e edema da parede intestinal podem causar obstrução intestinal, e infarto e perfuração intestinal podem ser complicações subsequientes. Se a intussuscepção progredir e não for adequadamente tratada pode tornar-se fatal. A causa desta patologia pode ser idiopática ou resultante de uma lesão orgânica pré-existente, como divertículos de Meckel, pólipos, hematomas, cistos, neurofibromas ou doenças malignas ${ }^{(1)}$. Esta patologia é relativamente comum em crianças, sendo geralmente idiopática, diferentemente do que é evidenciado em adolescentes e adultos, que apresentam uma causa orgânica comprovada na maioria dos casos. A intussuscepção é rara em adultos, e representa $5 \%$ de todas intussuscepções ${ }^{(1)}$.

Em adolescentes, a intussuscepção possui uma apresentação similar a crianças, mas freqüentemente

Trabalho realizado no Departamento de Cirurgia Geral do Hospital Universitário da Universidade de São Paulo, Universidade de São Paulo - SP. 
tem uma patologia de base, como observado em adultos $^{1}$. A intussuscepção é comumente negligenciada, apresentando um diagnostico pré-operatório correto em $32 \%$ dos $\operatorname{casos}^{(2)}$.

Embora uma origem maligna de intussuscepção possa ser descoberta em metade dos casos de adultos, o linfoma intestinal é uma causa incomum de intussuscepção em adolescentes e adultos (2).

\section{RELATO DE CASO}

Paciente 16 anos, masculino, previamente hígido procurou nosso serviço com história de dor súbita e intensa em quadrante inferior do abdome há 36 horas, associada a vômitos e fezes com sangue vivo. Negava febre ou perda de peso, nunca havia sido submetido a procedimento cirúrgico, e não apresentava história familiar de doença inflamatória intestinal ou neoplasia intestinal maligna. Ao exame físico se apresentava levemente desidratado, afebril e não possuía linfonodos palpáveis. $\mathrm{O}$ abdome era inocente, exceto por uma massa palpável no quadrante inferior direito. $\mathrm{O}$ exame proctológico revelava fezes com sangue.

O hemograma era normal e a radiografia simples de abdome demonstrou dilatação do intestino delgado sugestiva de obstrução parcial. A ultra-sonografia abdominal revelou espessamento focal da parede do ceco com uma estrutura tubular em seu interior. A colonoscopia demonstrou uma massa protuberante proveniente do orifício da válvula ileocecal (Figura 1). O segmento intussusceptado foi reduzido, e o paciente apresentou alívio completo dos sintomas, sendo então encaminhado para acompanhamento e investigação ambulatorial.

Após três semanas, o paciente retornou ao nosso hospital com recorrência dos sintomas há 24 horas. A ultra-sonografia abdominal revelou uma lesão em alvo no quadrante inferior direito, compatível com intussuscepção ileocecal. Devido à persistência dos sintomas, receio de uma nova intussuscepção e a possibilidade de compressão vascular mesentérica, o paciente foi submetido à cirurgia..

A laparotomia foi realizada, e uma massa polipóide foi evidenciada no íleo terminal com intussuscepção para o interior do ceco. Diversos linfonodos aumentados foram evidenciados no mesentério. O fígado e o baço não apresentavam al- terações, e disseminação peritoneal não foi evidenciada. Uma hemicolectomia direita estendida até os 20 $\mathrm{cm}$ distais do íleo com dissecção linfonodal e anastomose látero-lateral ileocólica foi realizada. Tentativas de reduzir a intussuscepção não foram realizadas.

O exame patológico da peça revelou uma massa polipóide de $4,5 \mathrm{~cm}$ oriunda do íleo distal (Figura 2), com aparência de carne de peixe e com infiltração da parede intestinal desde a mucosa até a subserosa e envolvimento de 6 linfonodos mesentéricos de um total de 18 linfonodos ressecados. A histologia revelou células de tamanho médio com padrão monótono difuso de infiltração, com muitas figuras mitóticas e células apoptóticas e numerosos macrófagos benignos com padrão de "céu estrelado" (Figura 3). No estudo imuno-histoquímico, CD-20, CD-10, Ki-67 (70\%) foram positivos e CD3, CD-5, BCL-2, EBV e CD-99 foram negativos. O diagnóstico final foi um linfoma de Burkitt de íleo distal (variante clínica esporádica) estágio II R (doença abdominal ressecada completamente).

Para o estadiamento tumoral foram realizadas tomografia computadorizada de crânio, tórax, abdome e pelve, que não evidenciaram doença metastática. A aspiração de medula óssea e biópsia se revelaram normais. Sorologias para HIV, IgG e IgM para vírus Epstein Barr e testes sorológicos para hepatites B e C,

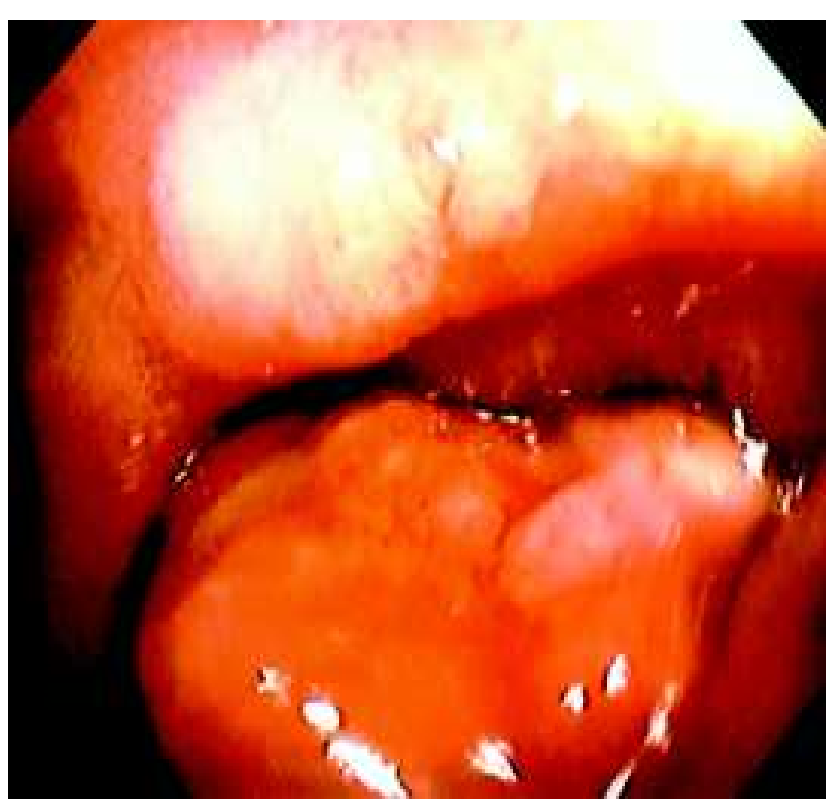

Figura 1 - Colonoscopia revelando uma massa protuberante proveniente do orifício da válvula ileocecal. 


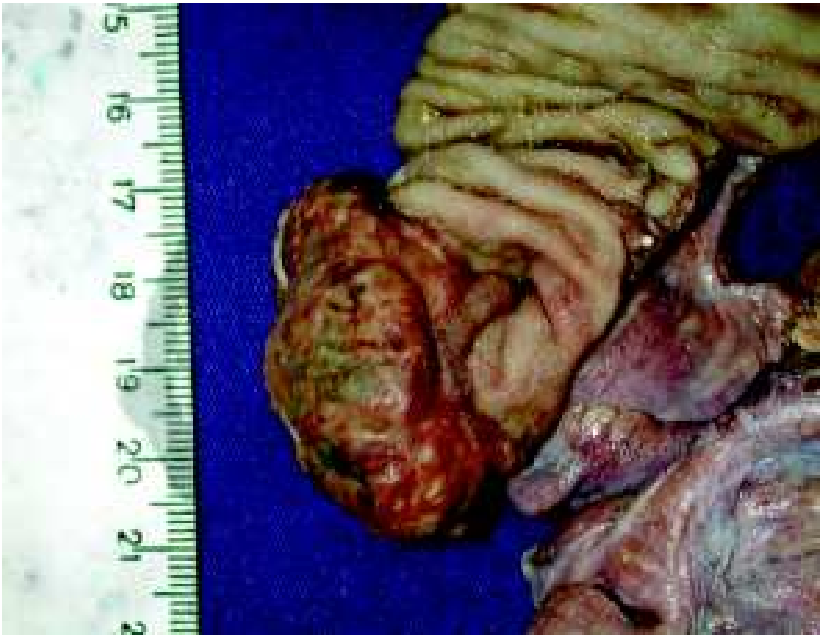

Figura 2 - Exame patológico revelando uma massa polipóide de $4,5 \mathrm{~cm}$ oriunda do íleo distal.

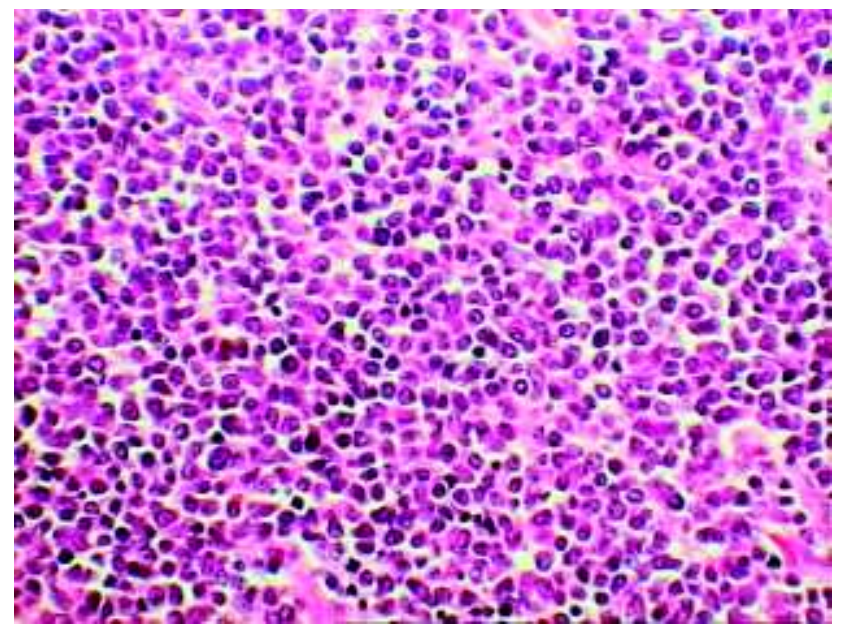

Figura 3 - Histologia demonstrando numerosos macrófagos benignos com padrão de "céu estrelado".

toxoplasmose e sífilis foram negativos. Desidrogenase lactato (DHL) era normal.

Uma vez que havia infiltração dos linfonodos ressecados por células tumorais oriundas do tumor intussusceptado ileal, e não se identificou outros lugares envolvidos pelo linfoma, o diagnóstico de um linfoma ileal primário foi fechado.

A recuperação pós-operatória transcorreu sem intercorrências. $\mathrm{O}$ paciente recebeu alta no segundo dia do pós-operatório, sendo encaminhado para quimioterapia adjuvante.

\section{DISCUSSÃO}

A intussuscepção em adolescentes é patologia rara, e a maioria das informações é proveniente de relatos de caso. Portanto, o manejo de intussuscepção em adolescentes deve ser similar ao de adultos. Enquanto em lactentes e crianças jovens $95 \%$ das intussuscepções são idiopáticas, em adolescentes e adultos uma lesão orgânica é geralmente identificada em cerca de $80 \%$ dos casos. Quarenta e seis por cento dos pacientes adultos têm uma doença maligna subjacente. Em adultos, a causa mais comum de intussuscepção entérica benigna são as aderências pósoperatórias, enquanto lesões malignas causando intussuscepção consistem essencialmente de doença metastática ${ }^{(2,3)}$.

A ocorrência de linfomas primários em intestino delgado é rara e representam menos de $2 \%$ de todas as neoplasias intestinais e $10-20 \%$ das neoplasias do intestino delgado. O íleo é o local mais comum, representando $50 \%$ dos linfomas do intestino delgado. A imunossupressão relacionada ao HIV predispõe ao desenvolvimento de linfomas não-Hodgkin (LNH) e deve ser excluída em todos os casos ${ }^{(4)}$.

Linfomas são o terceiro câncer mais comum em crianças e adolescentes nos Estados Unidos, correspondendo por aproximadamente $13 \%$ dos novos casos diagnosticados nesta faixa etária. LNH representam $60 \%$ dos linfomas nesta população. O linfoma de Burkitt é um linfoma de células B germinativo altamente agressivo, freqüentemente apresentando sítios extranodais. Translocação do gene myc no cromossomo 8q24 é uma característica genética constante e o vírus Epstein-Barr (EBV) é encontrado em uma proporção variável de casos. Três variantes clínicas são reconhecidas: endêmica, esporádica e associada à imunodeficiência. No linfoma de Burkitt esporádico, a freqüência de associação com EBV é inferior a 30\%. Casos esporádicos de linfoma de Burkitt tipicamente envolvem o abdome ou a cabeça e o pescoço. Tumores abdominais freqüentemente se apresentam com intussuscepção, como em nosso caso ${ }^{(5)}$.

Os sintomas predominantes da intussuscepção são aqueles da obstrução intestinal. O paciente se apresenta com náuseas, vômitos e dor abdominal. Melena ou fezes em geléia de morango estão presentes em apenas $29 \%$ dos casos. Febre, perda de peso, constipação, diarréia ou massa abdominal palpável são raras ${ }^{(2,3)}$.

Grupta et al ${ }^{(6)}$ recentemente, em 2007, publicaram uma casuística de 189 pacientes pediátricos com linfoma de Burkitt abdominal, tendo 33 (17,5\%) destes se apresentado com intussuscepção. A idade média ao diagnóstico foi 10 (3 a 19) anos, e a maioria dos paci- 
entes se apresentou dor abdominal (88\%), náuseas e/ ou vômitos $(42 \%)$.

A radiografia simples de abdome não é útil para o diagnóstico de intussuscepção. Na maioria dos lactentes e crianças jovens a redução da intussuscepção pode ser tentada com enema. Entretanto, em adultos, a redução não é freqüentemente sugerida como o tratamento da intussuscepção de etiologia incerta, devido ao alto risco de malignidade como causa da intussuscepção, e também devido a possibilidade de disseminação tumoral durante a manipulação ${ }^{(7,8)}$.

Exames mais acurados para o diagnóstico são a tomografia computadorizada e a ultra-sonografia, com uma sensibilidade de até $98,5 \%$ dos casos ${ }^{(2,3)}$. A ultrasonografia é fácil de realizar, mais reprodutível e menos invasiva do que outros métodos. Os achados clássicos de intussuscepção incluem os sinais em "alvo" ou "donut" na visão transversa e o sinal do "pseudorim" na visão longitudinal ${ }^{(9,10)}$. A maior limitação da ultra-sonografia para a avaliação de abdome agudo é a presença de ar no intestino, que leva a uma pobre transmissão e dificulta a interpretação da imagem. A tomografia computadorizada exibe achados semelhantes à ultra-sonografia, mas é mais cara e menos disponível em situações de emergência ${ }^{(11,12)}$. A colonoscopia é também uma ferramenta útil para a avaliação da intussuscepção e, como em nosso caso, pode ser usada como abordagem inicial na obstrução intestinal, tentando evitar uma cirurgia de emergência com o paciente em más condições clínicas e permitindo uma melhor investigação da causa da intussuscepção.

Brichon et al ${ }^{(13)}$ publicaram uma série de casos com 8 pacientes pediátricos com linfoma de Burkitt que se apresentaram com intussuscepção, e concluíram que a ultra-sonografia é o exame mais eficiente para diagnóstico de intussuscepção intestinal e algumas vezes da lesão primária. Neste trabalho, os auto- res ainda afirmam que se o linfoma não for visualizado pela ultra-sonografia, a laparotomia é necessária para o diagnóstico e eventual ressecção.

O tratamento padrão da intussuscepção em adultos não é um consenso universal. A intussuscepção em adolescentes e adultos pode ser tratada cirurgicamente de acordo com a condição subjacente mais provável (maligna ou benigna). Pacientes adolescentes e adultos com lesões entéricas que não tenham tido uma laparotomia prévia devem ser submetidos à ressecção sem redução, por causa da alta incidência de malignidade associada ${ }^{(2)}$. Isto pode ser realizado utilizando técnicas laparoscópicas, com os benefícios de um bom resultado cosmético, rápida recuperação e menos complicações ${ }^{(14)}$. Em nosso caso, nós preferimos uma laparotomia devido à possibilidade de compressão mesentérica vascular.

A cirurgia tem um papel limitado no tratamento, e é indicada em casos onde a ressecção completa pode ser realizada ou complicações do tumor estão presentes, como obstrução intestinal ${ }^{15}$. A quimioterapia varia de acordo com estágio da doença. Em estágio II $\mathrm{R}$, como em nosso caso, as taxas de sobrevida alcançam de 85 a $95 \%$ em 5 anos $^{5}$.

Abbasolu et al ${ }^{16}$ publicaram sua experiência com 40 casos de linfoma de Burkitt abdominal em pacientes entre 3 e 12 anos seguidos por mais de 10 anos. Em 10 casos o procedimento cirúrgico foi o tratamento de escolha, todos devidos a abdome agudos (5 obstruções intestinais, 3 intussuscepções, uma perfuração e uma apendicite aguda), enquanto os demais pacientes foram encaminhados para tratamento clínico. Os autores concluíram que a cirurgia deve ser reservada apenas para pacientes com doença localizada, devendo os pacientes com doença extensa intraabdominal terem seus procedimentos limitados à biópsia. Segundo os autores todos devem ser submetidos à quimioterapia.

\footnotetext{
ABSTRACT: Introduction: Intussusception occurs when a proximal segment of bowel telescopes into the lumen of the adjacent distal segment. It is common in children being usually idiophatic, in contrast to adolescents and adults, who have a demonstrable etiology in most cases. Intestinal lymphoma is an unusual cause of intussusception in adolescents and adults. Case Report: A 16 year-old male presented with right lower quadrant abdominal pain lasting for $\mathbf{3 6}$ hours, with vomiting and bloody stool. Physical examination was unremarkable except for a palpable mass at the right lower quadrant. Abdominal sonography revealed ileocecal intussusception. Colonoscopy showed a protruding mass from the orifice of ileocecal valve which was reduced, and the patient had full withdrawal of symptoms. Three weeks later, the patient came back to our hospital with recurrent symptoms. Laparotomy was performed and a polypoid mass was noted at the terminal ileum with intussusception into the cecum. A right hemicolectomy was performed. After pathologic examination and tumor staging, a primary Burkitt lymphoma of the distal ileum was diagnosed. Postoperative convalescence was uneventful and the patient was referred to adjuvant chemotherapy.
}

Key words: Lymphoma. Intussusception. Ileum. Immunohistochemistry. Neoplasms. 


\section{REFERÊNCIAS}

1. Huppertz HI, Soriano-Gabarro M, Grimprel E, Franco E, Mezner Z, Desselberger U, et al. Intussusception among young children in Europe. Pediatr Inf Dis J. 25 (1 Suppl):S22-9, 2006.

2. Azar T, Berger DL. Adult intussusception. Ann Surg. 1997; 226 (2): 134-8.

3. Yalamarthi S, Smith RC. Adult intussusception: case reports and review of literature. Postgrad Med J. 2005; 81 (953): 174-7.

4. Yahchouchy E, Etienne JC, Oberlin P, Fingerhut A. Double ileocaecal and colonic intussusception due to malignant lymphoma of the caecum in an HIV-positive patient. ANZ J Surg. 2001; 71 (3): 194-6.

5. Armitaje JO. Treatment of non-Hodgkin's lymphoma. New Engl J Med. 1993; 328(14): 1023-30.

6. Gupta H, Davidoff AM, Pui CH, Shochat SJ, Sandlund JT. Clinical implications and surgical management of intussusception in pediatric patients with Burkitt lymphoma. J Pediatr Surg. 42(6):998-1001; 2007.

7. Begos DG, Sandor A, Modlin IM. The diagnosis and management of adult intussusception. Am J Surg. 1997; 173: 88-94.

8. Khurrum BM, Hussain S, Wise M, Wexner SD. Controversy in the treatment of adult long ileocolic intussusception: case report. Am Surg. 2000; 66(8): 742-3.

9. Cerro P, Magrini L, Porcari P, De Angelis O. Sonographic diagnosis of intussusception in adults. Abdom Imaging. 2000; 25(1): 45-7.
10. Weissberg D, Schuble W, Leopold GR. Ultrasonographic appearance of adult intussusception. Radiol. 1977; 124(3): 791-2.

11. Gayer G, Zissen R, Apter S, Papa M, Hertz M. Pictorial review: adult intussusception- a CT diagnosis. Br J Radiol. 2002; 75: 185-90.

12. Warshauer DM, Lee JK. Adult intussusception at CT or MR imaging: clinical-imaging correlation. Radiol. 1999; 212(3): 85360.

13. Brichon P. Bertrand Y. Plantaz D. Burkitt's lymphoma revealed by acute intussusception in children. An Chir. 126(7):649-53, 2001.

14. Chiu CC, Wei PL, Huang MT, Wang W, Chen TC, Lee WJ. Laparoscopic treatment of ileocecal intussusception caused by primary ileal lymphoma. Surg Laparosc Endosc Percuth Tech. 2004; 14(2): 93-5

15. Stovroff MC, Coran AG, Hutchinson RJ. The role of surgery in American Burkitt's lymphoma in children. J Pediatr Surg. 1991; 26 (10): 1235-8.

16. Abbasolu L. Gun F. Salman FT. Celik A. Unuvar A. Gorgun O. The role of surgery in intraabdominal Burkitt's lymphoma in children. Euro J Pediatr Surg. 13(4):236-9, 2003.

\section{Endereço para correspondência:} ROBERTO IGLESIAS LOPES

Rua Baronesa de Itu, 721, apto. 121. Higienópolis

São Paulo - SP

CEP: 01231-001

Telefone: (0xx11) 3666-8291; Fax (0xx11) 3666-8266

E-mail: robertoiglesias@ terra.com.br 